\title{
Novel standoff detection system for the classification of chemical and biological hazardous substances combining temporal and spectral laser induced fluorescence techniques
}

\author{
Florian Gebert $^{1^{*}}$, Marian Kraus ${ }^{1}$, Lea Fellner ${ }^{1}$, Arne Walter ${ }^{1}$, Carsten Pargmann ${ }^{1}$, \\ Karin Grünewald ${ }^{1}$ and Frank Duschek ${ }^{1}$ \\ ${ }^{1}$ German Aerospace Center, Institute of Technical Physics, Langer Grund, Lampoldshausen, \\ 74239 Hardthausen, Germany \\ florian.gebert@dlr.de
}

\begin{abstract}
In an effort to reduce the potential risk of human exposure to chemical and biological hazardous materials, the demand increases for a detection system which rapidly identifies possible threats from a distance to avoid direct human contact to these materials. In this scope, we present a novel detection system which is able to measure simultaneously spectrally and temporally resolved laser induced fluorescence (LIF) signals excited by two consecutive laser pulses with different central wavelengths at $266 \mathrm{~nm}$ and $355 \mathrm{~nm}$. As shown in this paper, the setup enables fast data acquisition that provides a complete dataset in less than a few milliseconds at repetition rates of $100 \mathrm{~Hz}$. Furthermore, with its modular design it can be transported easily for operation at different locations. First measurements indicate a high performance with an accuracy of more than $97 \%$ in the distinguishability of bacterial specimen within a limited set of three representative bacterial species, namely Bacillus thuringiensis, Micrococcus luteus and Oligella urethralis. Together with the consecutive classification procedure, the setup promises to become a valuable tool for standoff detection of bio-hazards.
\end{abstract}

Keywords: Multispectral laser induced fluorescence, Fluorescence lifetime measurement, Standoff detection, Classification

\section{Introduction}

There are different scenarios, such as industrial accidents or terrorist attacks, in which people may get in contact with chemical or biological hazardous materials $[1,2]$. Depending on these scenarios the harmful substance can take different routes to humans, e.g. water supplies or as aerosol clouds. To be able to quickly take steps against the negative consequences on humans a fast standoff detection and identification system for sources of biological agents is essential. Different optical techniques can be used as a basis for such a detector and some of them are reviewed in ref. [3, 4]. 
One of the most widely used techniques for standoff detection of biological and chemical samples is laser-induced fluorescence (LIF) spectroscopy, since it provides high signal strength and thereby fast detection times on the order of seconds to minutes and long detection ranges up to a kilometer [5-9]. For aerosol samples, LIF spectroscopy is often combined with light detection and ranging (LIDAR) by making use of photon arrival time measurements $[3,7,9]$. These techniques are able to localize a thread quickly and map the spread of clouds and its propagation, as has been shown in different field trials. Depending on the scenario a promising approach is to combine standoff detectors with point sensors to increase the detection confidence [10].

In this paper we present a novel setup combining temporal and spectral LIF techniques to enhance the specificity of the classification algorithm used to distinguish bacterial samples. For spectrally resolved LIF the rather broad spectral features of the specimens of interest allow for a downscaling of the spectroscopic resolution from 1024 spectral channels in ref. [5] to 32 spectral channels with no reduction of the classification performance as presented in ref. [11]. But, in comparison to other techniques the rather low information content provides limited identification prospects. Laser pulses with different central wavelengths can be used to excite different fluorophores [5, 12], e.g. the amino acids tryptophan and tyrosine, using a laser wavelength of i.a. $266 \mathrm{~nm}$, or molecules present in metabolism of living cells like reduced nicotinamide adenine dinucleotide (NADH) and flavin adenine dinucleotide (FAD), using a laser wavelength of i.a. $355 \mathrm{~nm}$. The diverse spectral shapes of the fluorescence signals after excitation with these different laser pulses provide distinct information for the subsequent classification procedure. By combining the datasets of both excitation processes the classification performance can be improved significantly [5, 11, 13]. To further enhance the specificity in the presented setup we simultaneously acquire temporally resolved LIF signals for each excitation pulse using fast electronics. Since the fluorescence temporal behavior depends on the involved constituents and especially their microenvironments it can vary even between substances with similar chemical composition $[12,14,15]$. As we show below this variation provides enough diversity for a discrimination of three chosen bacterial suspensions excited and measured at a standoff distance of $3.5 \mathrm{~m}$ with an accuracy of $98 \%$. The distinguishability of more different bacterial samples will be investigated in the future. To the best of the author's knowledge this represents the first published use of temporally resolved fluorescence data from bacterial samples measured at standoff distances applied to a subsequent classification. The performance is compared to that using the simultaneously acquired spectrally resolved LIF data and a combination of both datasets.

\section{Experimental Setup}

A schematic view of the setup is displayed in Fig. 1. A Nd:YAG laser system (Innolas Picolo Magna EVO III) that simultaneously provides laser pulses with four different center wavelengths with a repetition rate of $100 \mathrm{~Hz}$ is used. The pulse duration of all laser pulses is below $0.7 \mathrm{~ns}$. For LIF excitation we use the pulses in the ultraviolet 
spectral region around $266 \mathrm{~nm}$ and $355 \mathrm{~nm}$. After passing through a setup for intensity control the pulses with a center wavelength of $355 \mathrm{~nm}$ are temporally retarded using an optical delay line [16]. It consists of two almost parallel mirrors aligned at a small angle $\beta$ between each other, see Fig. 2.a). Therefore, the reflection angles $\alpha_{i}$ of the laser beam path become smaller for each consecutive reflection, so that after a certain number of reflections, depending on the tilt angle $\beta$, the laser pulse changes its propagation direction and returns to the side of the delay line where it has entered. This way, the extent of the mirrors can be used twice, reducing the size of the setup. After spatially overlapping the pathway of the laser pulses with center wavelengths of $266 \mathrm{~nm}$ and $355 \mathrm{~nm}$ they are sent to the sample.

The combined beam path is guided to a fast photodiode and the resulting measurements are displayed in Fig. 2.b). A delay of up to $120 \mathrm{~ns}$ is achieved, corresponding to a travelled distance of $32 \mathrm{~m}$ with a mirror spacing $\mathrm{d}$ of approximately $0.8 \mathrm{~m}$. In the experiment a delay of approximately $100 \mathrm{~ns}$ between the two pulses and a repetition frequency of this pulse train of $100 \mathrm{~Hz}$ are used.

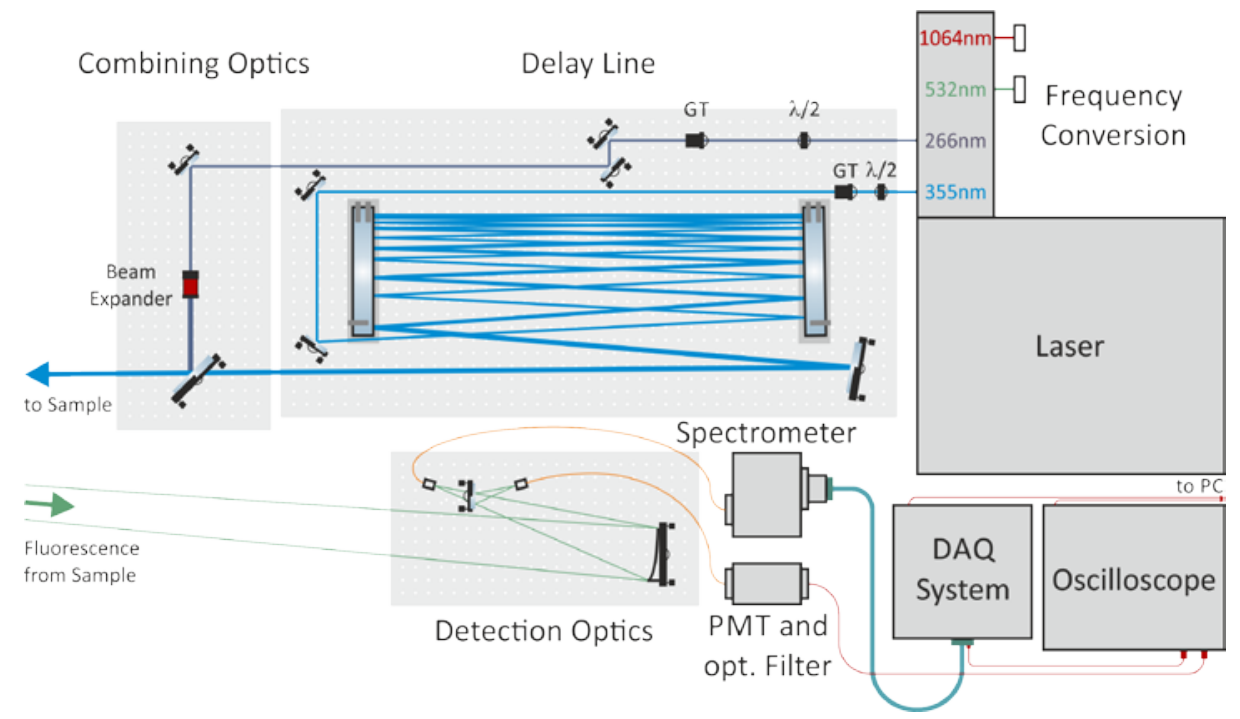

Fig. 1. Experimental setup: a Nd:YAG Laser simultaneously provides laser pulses with two different center wavelengths in the ultraviolet spectral region. After retarding the $355 \mathrm{~nm}$ pulses, their beam path is overlapped with the one of the laser pulses with a wavelength of $266 \mathrm{~nm}$ and sent to the sample. The fluorescence response is measured using a setup consisting of optical filters and photo multiplying tubes (PMT) and a spectrometer containing a PMT-array. Afterwards, the electronical signals are read out using a custom-made data acquisition system and a fast oscilloscope. ( $\lambda / 2$ : half-wave plate; GT: Glan-Taylor prism) 
a)

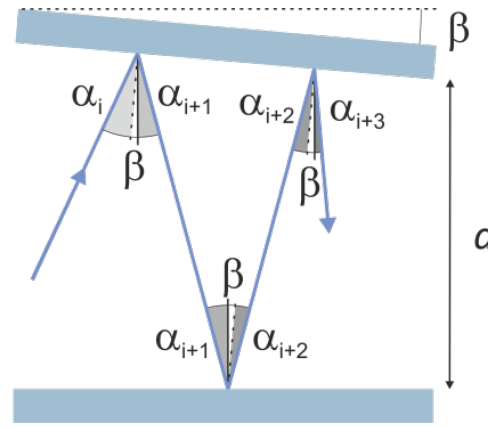

b)

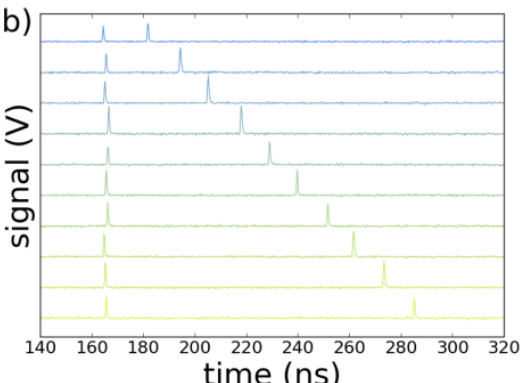

time (ns)

Fig. 2. a) Schematic view of the optical delay line, b) Delay of two consecutive pulses with different central wavelengths measured using a fast photodiode: from top to bottom the angle $\beta$ was reduced to allow four additional reflections for each consecutive measurement.

The fluorescence response is focused into two fiber bundles with a diameter of approx. $2 \mathrm{~mm}$ using an off-axis parabolic mirror with a diameter of $101.6 \mathrm{~mm}$. One of the bundles guides the radiation to a compact grating-based spectrometer (Hamamatsu A10766) combined with a 32 channel photo multiplying tube (PMT) array (Hamamatsu H7260). The electronical signals are read out by a customized data acquisition system (Vertilon Photoniq IQSP482). To be able to collect the spectral fluorescence data of the consecutive $266 \mathrm{~nm}$ and $355 \mathrm{~nm}$ laser pulses the data acquisition system provides a fast internal gating. In simplified terms, it consists of two banks and each bank is connected to the 32 PMT channels. After an adjustable time, corresponding to the time it takes for the radiation to travel from the sample to the detector, the first bank is triggered and starts to integrate for $50 \mathrm{~ns}$. After a time delay, constituted by the delay of the two consecutive laser pulses, the second bank is triggered and integrates the fluorescence signals of the second excitation also for $50 \mathrm{~ns}$. The second fiber bundle guides the radiation to a setup consisting of optical filters and PMTs which are connected to a fast Oscilloscope (Keysight DSO-X 6004a) recording the temporal fluorescence data with a resolution of down to $0.05 \mathrm{~ns}$. The time base of the data acquisition is a trigger pulse generated by the laser control electronics. The data acquisition parameters are controlled via an in-house developed LabVIEW [17] program.

\section{$3 \quad$ Experimental Results and Discussion}

The identification prospects using our setup for bacterial samples were investigated by measurements on three different bacterial suspensions. The measurements were performed at a distance of $3.5 \mathrm{~m}$ and with optical pulse energies of about $30 \mu \mathrm{J}$ and $50 \mu \mathrm{J}$ for laser pulses at $266 \mathrm{~nm}$ and $355 \mathrm{~nm}$, respectively. Bacteria were cultivated on nutrient agar 1 (Sifin, Berlin, Germany) at $37^{\circ} \mathrm{C}$ for $24 \mathrm{~h}$. Afterwards, the colony material was harvested, suspended and diluted in phosphate buffered saline (PBS) to reach a concentration of the order of $10^{8}$ to $10^{9}$ colony forming units per ml. For more detail on the sample preparation the interested reader is referred to [5].The measure- 
ments were conducted within two hours after preparation, since diminishing nutrient supply can have an influence on the spectral shapes of bacterial samples as investigated in ref. [18].

For each of the three samples 100 consecutive single excitation spectra and their mean are displayed in Fig. 3.a) and b). For the excitation at energies corresponding to a wavelength of $266 \mathrm{~nm}$ the spectral features of Bacillus thuringiensis and Micrococcus luteus are almost similar making an unambiguous assignment impossible, but the data for Oligella urethralis shows a distinct spectral behavior, see Fig 3.a). For the excitation at $355 \mathrm{~nm}$ (see Fig 3.b)), the distinguishability of the fluorescence signal of the three samples is more obvious. But, by using the complete spectral dataset the specificity of the classification is enhanced and thereby an almost clear assignment of the bacterial sample is possible.
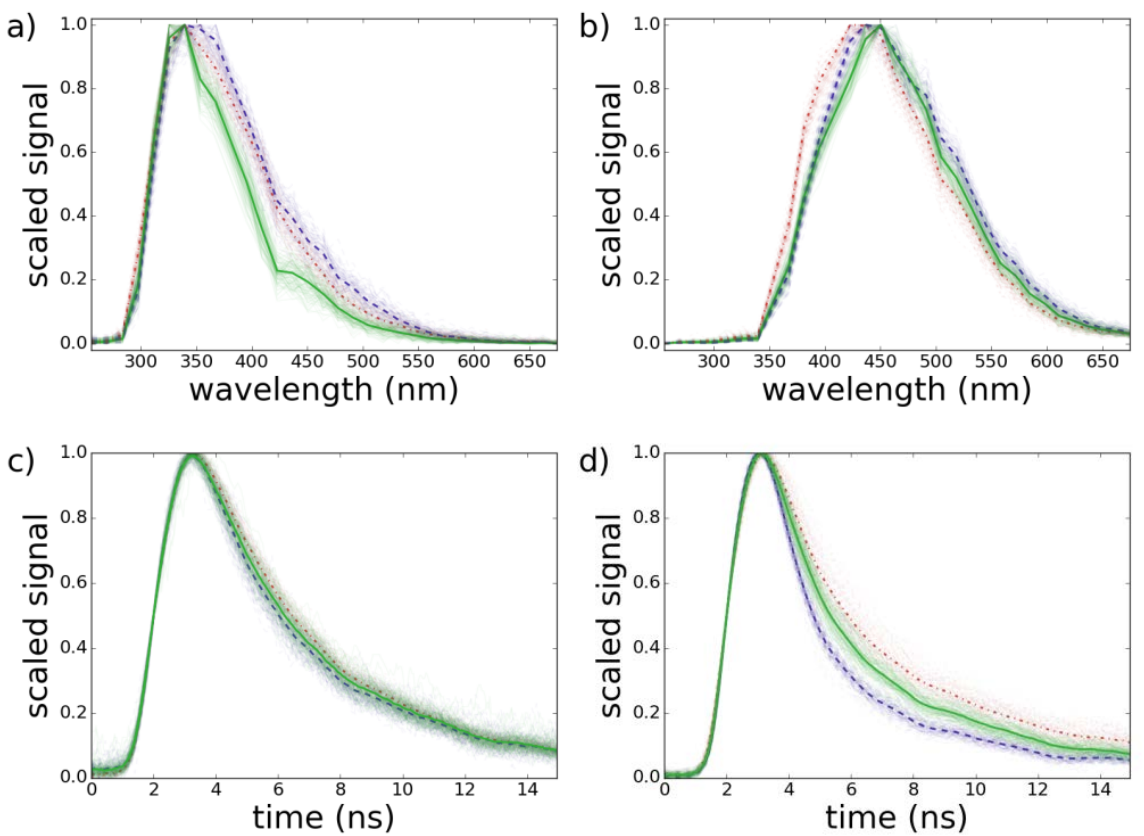

Fig. 3. Spectrally a), b) and temporally c), d) resolved LIF signals after excitation with laser pulses with wavelengths of $266 \mathrm{~nm}$ a), c) and $355 \mathrm{~nm} \mathrm{~b}$ ), d). To Obtain the temporally resolved LIF signals optical bandpass filters around $310 \mathrm{~nm} \mathrm{c}$ ) and $460 \mathrm{~nm} \mathrm{~d}$ ) are used. For better visibility of the differences within the datasets the opaque lines display the mean of 100 spectra acquired within $1 \mathrm{~s}$ and the transparent lines are 100 single excitation spectra to illustrate shotto-shot variations. Dash dotted, red: Bacillus thuringiensis; dashed, blue: Micrococcus luteus; solid, green: Oligella urethralis.

In Fig. 3.c) and d) the corresponding temporally resolved fluorescence data is displayed. For the measurements, different optical filters at $310 \mathrm{~nm}$ and $460 \mathrm{~nm}$ with an optical bandwidth of $10 \mathrm{~nm}$ are placed in front of the PMTs. The time dependent fluorescence signal of the different samples excited at $266 \mathrm{~nm}$ and measured at $310 \mathrm{~nm}$ 
cannot be distinguished from each other. For the $355 \mathrm{~nm}$ excitation the time signals show distinguishable shapes.

The identification performance of the system is evaluated using the C5.0 decision tree algorithm. A dataset comprised of 500 single-shot spectral and temporal LIF measurements for each bacterial sample was analyzed. Within the dataset $75 \%$ of the data was used for training and $25 \%$ for testing. The results of the classification are displayed in Table 1.a)-c).

Table 1. Classification results using a) the spectral LIF data, b) the temporal LIF data and c) the combined dataset. Columns: predictions; rows: reference.
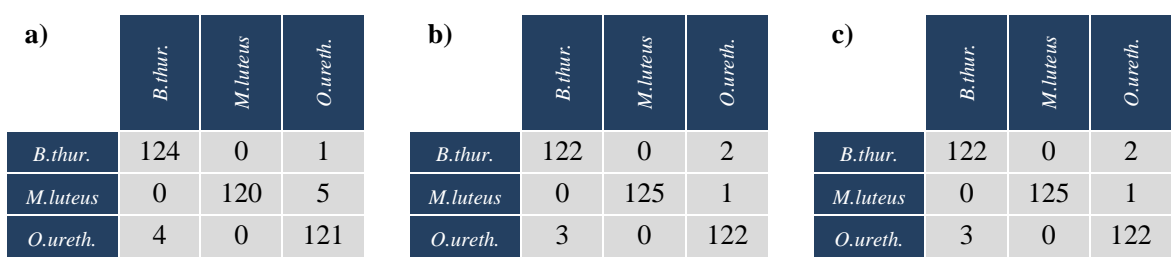

For the spectral data an accuracy of $97 \%$ is achieved whereas for the temporal data the accuracy is $98 \%$. For the combined dataset the accuracy amounts to $98 \%$, indicating that the fluorescence decay determines the performance of the classification using the combined dataset for the three chosen samples.

We also performed measurements on a larger set of bacterial samples, but until now strong variations of the fluorescence intensities for the used bacterial concentrations do not allow an automated classification. To prevent saturation of the detection system for all samples the laser pulse energies were fixed so that the system does not saturate for the sample with the largest fluorescence signal. The resulting low intensity of some temporal measurements for other samples leads to signals contaminated by electronic noise, making a large part of the dataset unusable for the classification algorithm. The spectral data acquisition is less demanding and the complete dataset was used for an identification of seven bacterial samples as reported in ref. [11]. An improved algorithm that only considers the temporal data fulfilling certain intensity criteria could be used to enhance the performance of the spectral assignment. In another approach an automated power adjustment may be added to stay in the dynamic range of the detection system.

\section{$4 \quad$ Summary and Outlook}

This paper presents a novel detection system that is able to simultaneously record temporal and spectral LIF data at standoff distances of $3.5 \mathrm{~m}$ with a data acquisition rate of $100 \mathrm{~Hz}$. The system provides 32 spectral channels and a temporal resolution down to $0.05 \mathrm{~ns}$. Using this setup we performed measurements on three different bacterial species, namely Bacillus thuringiensis, Micrococcus luteus and Oligella urethralis. The data permits an automated assignment of single shot spectra with an accuracy of $98 \%$, utilizing the C5.0 decision tree algorithm. 
For the small dataset of three samples the temporal LIF data shows a good distinguishability, even for similar structured samples like different bacterial species, and can enhance the performance of the classification. Therefore, we will investigate new strategies to incorporate the temporal fluorescence signals in the classification process. Additionally, more elaborate algorithms are investigated to enhance the classification performance.

Altogether, we presented a novel setup combining spectral and temporal data acquisition. First measurements and the subsequent classification show that the identification of different specimen within a dataset of three different bacterial species is possible using the temporal LIF data. Using novel acquisition and classification strategies the presented setup has the potential to become a valuable tool to provide additional information for standoff detection of biological hazards.

\section{References}

1. Burrows, W.D., Renner, S.E.: Biological warfare agents as threats to potable water. Environmental Health Perspectives 107, 975-984 (1999).

2. Henderson, D.A.: The looming threat of bioterrorism. Science 283, 1279-1282 (1999).

3. Buteau, S., Ho, J., Lahaie, P., Rowsell, S., Simard, J.-R., Kadlcak, J., Sgarzi, G., Tonini, P., Heuvel, J.C. van den, Blatny, J.M., Farsund, Ø., Gran, H.-C., S.tianLovold, Rustad, G., Sletmoen, M., Baxter, K., Castle, M., Foot, E.V.J., Swim, C., Vanderbeek, R.: Laser based standoff detection of biological agents. North Atlantic Treaty Organisation, Research and Technology Organisation (2010).

4. Gaudio, P., Gelfusa, M., Murari, A., Pizzoferrato, R., Carestia, M., Cenciarelli, O., Parracino, S., Ludovici, G., Gabriele, J., Gabbarini, V., Giovanni, D.D., Rossi, R., Ciparisse, J.F., Bellecci, C., Malizia, A.: Application of optical techniques to detect chemical and biological agents. In: Defence S\&T Technical Bulletin. pp. 1-13. Defence S\&T Technical Bulletin (2017).

5. Duschek, F., Fellner, L., Gebert, F., Grünewald, K., Köhntopp, A., Kraus, M., Mahnke, P., Pargmann, C., Tomaso, H., Walter, A.: Standoff detection and classification of bacteria by multispectral laser-induced fluorescence. Advanced Optical Technologies 6, 75-83 (2017).

6. Farsund, Ø., Rustad, G., Skogan, G.: Standoff detection of biological agents using laser induced fluorescence-a comparison of $294 \mathrm{~nm}$ and $355 \mathrm{~nm}$ excitation wavelengths. Biomedical Optics Express 3, 2964 (2012).

7. Jonsson, P., Elmqvist, M., Gustafsson, O., Kullander, F., Persson, R., Olofsson, G., Tjärnhage, T., Farsund, Ø., Haavardsholm, T.V., Rustad, G.: Evaluation of biological aerosol stand-off detection at a field trial. In: Carrano, J.C. and Collins, C.J. (eds.) Optically Based Biological and Chemical Detection for Defence V. SPIE 7484 (2009).

8. Sharma, R.C., Kumar, D., Kumar, S., Joshi, D., Srivastva, H.B.: Standoff Detection of Biomolecules by Ultraviolet Laser-Induced Fluorescence LIDAR. IEEE Sensors Journal 15, 3349-3352 (2015). 
9. Simard, J.R., Roy, G., Mathieu, P., Larochelle, V.: Standoff integrated bioaerosol active hyperspectral detection (Sindbahd): Final report. Defence R\&D Canada (2003).

10. Buteau, S., Rowsell, S.: Biological material detection identification and monitoring: Combining point and standoff sensors technologies. In: Burgess, D., Owen, G., Bouma, H., Carlysle-Davies, F., Stokes, R.J., and Yitzhaky, Y. (eds.) Optics and Photonics for Counterterrorism, Crime Fighting, and Defence XII. SPIE 9995 (2016).

11. Kraus, M., Fellner, L., Gebert, F., Grünewald, K., Pargmann, C., Walter, A., Duschek, F.: Comparison of Classification Models of Spectral Data of Laser Induced Fluorescence. In: 1st Scientific International Conference on CBRNe. Springer (2017).

12. Lakowicz, J.R.: Principles of Fluorescence Spectroscopy. Springer-Verlag New York Inc. (2006).

13. Sivaprakasam, V., Lin, H.-B., Huston, A.L., Eversole, J.D.: Spectral characterization of biological aerosol particles using two-wavelength excited laser-induced fluorescence and elastic scattering measurements. Optics Express 19, 6191 (2011).

14. Kinkennon, A.E., McGown, L.B.: Fluorescence lifetime characterization of bacteria using total lifetime distribution analysis with the maximum entropy method. Journal of Fluorescence 7, 201-210 (1997).

15. Awad, F., Ramprasath, C., Mathivanan, N., Aruna, P.R., Ganesan, S.: Steadystate and fluorescence lifetime spectroscopy for identification and classification of bacterial pathogens. Biomedical Spectroscopy and Imaging 3, 381-391 (2014).

16. Herriott, D.R., Schulte, H.J.: Folded Optical Delay Lines. Applied Optics 4, (1965).

17. Elliott, C., Vijayakumar, V., Zink, W., Hansen, R.: National Instruments LabVIEW: A Programming Environment for Laboratory Automation and Measurement. Journal of the Association for Laboratory Automation 12, 1724 (2007).

18. Fellner, L., Gebert, F., Walter, A., Grünewald, K., Duschek, F.: Variations in fluorescence spectra of a bacterial population during different growth phases. In: 1st Scientific International Conference on CBRNe. Springer (2017). 